Table 1 PTH-046

\begin{tabular}{lcc}
\hline \multicolumn{2}{c}{ Mean cytokine level \pm SEM $(\mathbf{p g} / \mathbf{m l})$} \\
\hline Cytokine & Rutgeert score $\leq 1$ & Rutgeert score $\geq 2$ \\
IL-2 & $2.4 \pm 0.1$ & $2.3 \pm 0.1$ \\
IL-4 & $0.3 \pm 0.2$ & $0.5 \pm 0.3$ \\
IL-6 & $2469.1 \pm 969.2$ & $5485.7 \pm 2020$ \\
IL-10 & $7.3 \pm 4.8$ & $4.6 \pm 0.8$ \\
TNF $\alpha$ & $1.6 \pm 0.1$ & $2.7 \pm 1.5$ \\
IFN $\gamma$ & $1.3 \pm 0.2$ & $6.6 \pm 2.1$ \\
IL-17a & $0.5 \pm 0.4$ & $2.5 \pm 0.7$ \\
\hline
\end{tabular}

were thiopurines 13, Infliximab 1 and nil 10. Endoscopic severity was i0 $n=5$, i1 $n=6, i 2 n=5$, i3 $n=3, i 4 n=5$. Mean cytokine concentrations from supernatants are shown in the table 1. Comparison between $\mathrm{RS} \leq 1$ and $\geq 2$ showed that pro-inflammatory cytokines IL-17a $(p<0.02)$ and IFN $\gamma(p<0.03)$ were significantly higher in RS i2-i4 neo terminal ileum as compared with those with RS i0-i1. The regulatory cytokine IL-10 was significantly higher in patients with $\mathrm{RS} \leq 1(\mathrm{p}<0.038)$.

Conclusion Cytokine profiles in those with $\mathrm{RS} \geq 2$, show higher levels of IL-17a and IFN $\gamma$ and reduced IL-10 compared to $\mathrm{RS} \leq 1$. This profile supports a Th17 and Th1 mediated response as one of the early instigators of endoscopic progression in postoperative $\mathrm{CD}$. The authors' observation is consistent with recent findings of a $\mathrm{T}$ cell subset able to produce cytokines involved in both Th1 and Th17 responses. Previous therapies directed at Th1 pathway, for example, anti-IL-12p40 antibody ustekinumab and anti-IFN $\gamma$ Fontolizumab failed to show significant clinical benefit in $\mathrm{CD}$. Given our findings targeting the Th17 response, for example, with anti-IL-23 antibodies and anti-IL-17 may deliver improved therapeutic outcome.

Competing interests None.

Keywords cytokines, postoperative Crohn's disease, TH1/ TH17.

\section{TH1/TH17 PROFILES IN CROHN'S DISEASE: A CROSS SECTIONAL SINGLE CENTRE STUDY IN POSTOPERATIVE CROHN'S DISEASE}

doi:10.1136/gut.2011.239301.447

A U Murugananthan, 1,* D Bernardo, ${ }^{1}$ P Tozer, ${ }^{1}$ C T Tee, ${ }^{1}$ E Mann, ${ }^{1}$ A L Hart, ${ }^{2}$ N Arebi, ${ }^{2}$ S C Knight, ${ }^{1} \mathrm{H} O$ Al-Hassi ${ }^{1}$ Antigen Presentation Research Group, Imperial College, London, UK; ${ }^{2}$ Gastroenterology, St Mark's Hospital, London, UK

Introduction Th1 and Th17 pathways are implicated in Crohn's disease (CD). In operative resection samples healthy ileum shows high TGF $\beta$ levels in patients who develop recurrence, with TGF $\beta$ being a known activator of the Th17 response. Other studies in CD show a dominant Th1 cytokine profile, with high levels of IFN $\gamma$, which reduce Th17 response and augment Th1 response. The relationship of Th1/Th17 cytokine profiles in postoperative $\mathrm{CD}$ has not been examined. The authors aimed to study tissue Th1/Th17 cytokine secretion after in vitro biopsy culture in postoperative CD.

Methods Colonoscopy was undertaken in postoperative CD patients. Recurrence graded as no/minimal inflammation (Rutgeert Score $(R S) \leq 1$ ) or progressive inflammation $(R S \geq 2$ ). Ileal biopsies were cultured overnight and cell free supernatants obtained. Supernatant cytokines (IL-2, IL-4, IL-10, IL-17 TNF $\alpha$, INFg and IL-6) were assessed by flow cytometry using cytometric bead array (Becton Dickinson). Statistical analysis was via unpaired t tests.

Results Consecutive patients attending endoscopy ( $\mathrm{n}=24$, $9 \mathrm{M} / 15 \mathrm{~F}$ ) were identified. Mean age 45.0 years and time from I to $C$ resection was 5.8 years; 5 patients were smokers. Drugs 